\title{
REPRESENTING AN ISOTONE MAP BETWEEN TWO BOUNDED ORDERED SETS BY PRINCIPAL LATTICE CONGRUENCES
}

\author{
GÁBOR CZÉDLI
}

\begin{abstract}
For bounded lattices $L_{1}$ and $L_{2}$, let $f: L_{1} \rightarrow L_{2}$ be a lattice homomorphism. Then the map $\operatorname{Princ}(f): \operatorname{Princ}\left(L_{1}\right) \rightarrow \operatorname{Princ}\left(L_{2}\right)$, defined by $\operatorname{con}(x, y) \mapsto \operatorname{con}(f(x), f(y))$, is a 0-preserving isotone map from the bounded ordered set $\operatorname{Princ}\left(L_{1}\right)$ of principal congruences of $L_{1}$ to that of $L_{2}$. We prove that every 0 -preserving isotone map between two bounded ordered sets can be represented in this way. Our result generalizes a 2016 result of G. Grätzer from $\{0,1\}$-preserving isotone maps to 0 -preserving isotone maps.
\end{abstract}

\section{INTRODUCTION AND OUR RESULT}

We assume that the reader has some familiarity with lattices and their congruences; if not then Grätzer [13, 19] and the freely down-loadable Nation [33] are recommended. Postponing some details about our motivation and a short survey of related results to Section 2, here we are going to get to our result in a short way.

For a lattice $L$, let $\operatorname{Princ}(L)=\langle\operatorname{Princ}(L) ; \subseteq\rangle$ denote the ordered set of principal congruences of $L$. A congruence of $L$ is principal if it is of the form $\operatorname{con}(a, b)=$ $\operatorname{con}_{L}(a, b)$ for some elements $a, b \in L$, that is, if it is generated by a single pair $\langle a, b\rangle$. If $L$ is bounded, which means that $0,1 \in L$, then so is $\operatorname{Princ}(L)$. In 2013, Grätzer [14] proved the converse: up to isomorphism, every bounded ordered set is of the form $\operatorname{Princ}(L)$ where $L$ is a bounded lattice. Since no similar characterization is known for non-bounded ordered sets in general, we study the representability of isotone maps by principal lattice congruences only among bounded ordered sets. For bounded lattices $L_{1}, L_{2}$ and a lattice homomorphism $g: L_{1} \rightarrow L_{2}$, it is natural to consider the map

$$
\begin{aligned}
\operatorname{Princ}(g): \operatorname{Princ}\left(L_{1}\right) & \rightarrow \operatorname{Princ}\left(L_{2}\right), \text { defined by } \\
\operatorname{con}_{L_{1}}(x, y) & \mapsto \operatorname{con}_{L_{2}}(g(x), g(y)) .
\end{aligned}
$$

It was observed by Grätzer [20] that (1.1) defines indeed a map, since one can easily show that $\operatorname{con}_{L_{1}}\left(x_{1}, y_{1}\right)=\operatorname{con}_{L_{1}}\left(x_{2}, y_{2}\right)$ implies that $\operatorname{con}_{L_{2}}\left(g\left(x_{1}\right), g\left(y_{1}\right)\right)=$ $\operatorname{con}_{L_{2}}\left(g\left(x_{2}\right), g\left(y_{2}\right)\right)$. Clearly, the map $\operatorname{Princ}(g)$ is 0 -preserving and isotone. The following definition is quite natural; analogous concepts have been used for (not necessarily principal) congruences in several earlier papers including Czédli [1] and Grätzer [20].

2000 Mathematics Subject Classification. 06B10, 18B05. Oct 8, 2017, revised April 30, 2018.

Key words and phrases. Principal congruence, lattice congruence, ordered set, poset, quasicolored lattice, preordering, quasiordering, isotone map, representation.

This research was supported by NFSR of Hungary (OTKA), grant number K 115518. 
Definition 1.1. Let $f: P_{1} \rightarrow P_{2}$ be a 0-preserving isotone map from an ordered set $P_{1}$ with 0 to an ordered set $P_{2}$ with 0 . We say that $f$ is representable by principal congruences of bounded lattices if there exist lattices $L_{1}$ and $L_{2}$, order isomorphisms $h_{i}: P_{i} \rightarrow \operatorname{Princ}\left(L_{i}\right)$, for $i \in\{1,2\}$, and a lattice homomorphism $g: L_{1} \rightarrow L_{2}$ such that $f=h_{2}^{-1} \circ \operatorname{Princ}(g) \circ h_{1}$, that is, the diagram

$$
\begin{array}{ccc}
\left\langle P_{1} ; \leq_{P_{1}}\right\rangle & f & \left\langle P_{2} ; \leq_{P_{2}}\right\rangle \\
h_{1} \downarrow & h_{2}^{-1 \uparrow} \\
\left\langle\operatorname{Princ}\left(L_{1}\right) ; \subseteq\right\rangle & \stackrel{\operatorname{Princ}(g)}{\longrightarrow}\left\langle\operatorname{Princ}\left(L_{2}\right) ; \subseteq\right\rangle
\end{array}
$$

is commutative. If we can find lattices $L_{1}$ and $L_{2}$ of lengths at most $m$ and $n$, respectively, such that (1.2) holds, then we say that $f$ is representable by principal congruences of lattices of lengths at most $m$ and $n$. We also say that the lattice homomorphism $g$ represents $f$ by means of principal congruences.

We say that $f$ in (1.2) is 0-separating, 1-preserving, and 0-preserving if we have that $\left\{x \in P_{1}: f(x)=0\right\}=\{0\}, f(1)=1$, and $f(0)=0$, respectively. Of course, the 1-preserving property assumes that both $P_{1}$ and $P_{2}$ have largest elements. It was proved in Czédli [3] that if $f$ has all the three properties listed above, then it is representable by principal congruences of bounded lattices. Later, Grätzer [20] proved that the first of the three conditions can be omitted, that is, whenever $f$ in (1.2) is 0-preserving and 1-preserving, then it is representable by principal congruences of bounded lattices. Strengthening this result even further, our aim is the prove that the preservation of 0 in itself guarantees representability; this is formulated in our theorem below.

Theorem 1.2. If $f: P_{1} \rightarrow P_{2}$ is a 0 -preserving isotone map from a bounded ordered set $P_{1}=\left\langle P_{1} ; \leq_{P_{1}}\right\rangle$ to a bounded ordered set $P_{2}=\left\langle P_{2} ; \leq_{P_{2}}\right\rangle$, then $f$ is representable by principal congruences of bounded lattices of lengths at most 5 and 7.

Theorem 1.2 gives an affirmative answer to F. Wehrung's question asked at the conference SSAOS-55, Nový Smokovec, Slovakia, 2017. Related results on ordered sets of principal congruences have recently been given in Czédli $[3,4,6,7,9,8]$, Grätzer [14, 20, 21, 22, 23], and Grätzer and Lakser [28, 29].

Remark 1.3. If none of $P_{1}$ and $P_{2}$ is a singleton, then we can chose $L_{1}$ and $L_{2}$ in Theorem 1.2 such that $L_{1}$ is of length 5 while $L_{2}$ is of length 7 .

Outline. Section 2 contains a mini survey of earlier results that motivate our present work. The rest of the paper is devoted to the proof of Theorem 1.2 and Remark 1.3. Section 3 describes the construction we need; first in a pictorial and easy-to-understand way for a concrete example, and then we expand this visual description to a general construction. Section 4 verifies our construction, whereby the theorem follows. Also, Section 4 points out why Remark 1.3 holds.

\section{Motivation AND A Mini SURVEY}

There are so many results on congruence lattices of lattices which motivate the present paper that this section, added on April 30, 2018, is restricted only to a mini survey of them. This short section and the list of the papers referenced here are far from being complete; a complete treatment would need a whole book. For much 
more extensive and very deep surveys up to their publication dates, the reader is referred to the monograph Grätzer [19] and to the book chapters Grätzer [16] and [17] and Wehrung [37], [38], and [39].

By a well-known old result of Funayama and Nakayama [12], the lattice $\operatorname{Con}(L)=$ $\langle\operatorname{Con}(L) ; \subseteq\rangle$ of all congruences of a lattice $L$ is distributive. The converse for the finite case is due to R. P. Dilworth but, independently, it was first published in Grätzer and Schmidt [30]. This result states that every finite distributive lattice is (isomorphic to) the congruence lattice $\operatorname{Con}(L)$ of a finite lattice $L$. In spite of several positive results, mile-stoned by Huhn [32] and Schmidt [35], which represent some infinite distributive algebraic lattices as congruence lattices of lattices; it was a real breakthrough when Wehrung [36] presented a distributive algebraic lattice $D$ such that $D \cong \operatorname{Con}(L)$ holds for no lattice $L$. Later, such a distributive algebraic lattice $D$ of minimal cardinality was given by Růžička [34].

Compared to the infinite case, much more results have been proved on the representability of finite distributive lattices $D$ by congruence lattices of finite lattices $L$. There are several results in which, in addition to $D \cong \operatorname{Con}(L)$, the lattice $L$ has some nice properties or its automorphism group is isomorphic to a given finite group; we mention Grätzer and Knapp [24] and Grätzer and Schmidt [31] as some attracting examples of this sort. A homomorphism $f: L_{1} \rightarrow L_{2}$ between two lattices naturally induces an isotone map from $\operatorname{Con}\left(L_{1}\right)$ to $\operatorname{Con}\left(L_{2}\right)$ or backwards, and various papers represent isotone maps between two finite distributive lattices in this way; see, for example, Grätzer and Lakser [25]. Several papers do this so that the lattices $L_{1}$ and $L_{2}$ have some nice properties; see, for example, Czédli [1] and Grätzer and Lakser [26] and [27]. Instead of representing a single map, there is a whole theory of representing families of isotone maps; see Wehrung [39].

In a pioneering paper, Grätzer [14] proved that every bounded ordered set $P=$ $\langle P ; \leq\rangle$ is isomorphic to $\langle\operatorname{Princ}(L) ; \subseteq\rangle$ for some lattice $L$. This result naturally leads to the following general problem: find the " $\langle P$, Princ $(L)\rangle$-type" counterparts of the " $\langle D, \operatorname{Con}(L)\rangle$-type" results mentioned so far in this section and, in addition, find analogous " $\langle P \subseteq D, \operatorname{Princ}(L) \subseteq \operatorname{Con}(L)\rangle$-type" representability results. Some concrete instances of this general problem are formulated at the end of Grätzer [17].

The present paper is motivated by and contributes to the progress outlined in this section above and mentioned right after Theorem 1.2. In spite of this progress, the present paper, and the very recent Czédli and Mureşan [11], we are far from the solution of the above-mentioned general problem.

\section{The CONSTRUCtion}

3.1. Decomposing $f$. Let $P_{1}$ and $P_{2}$ be bounded ordered sets. Assume that $f: P_{1} \rightarrow P_{2}$ is a 0 -preserving isotone map. Let $P_{3}$ be the principal ideal of $P_{2}$ generated by $f\left(1_{P_{1}}\right)$, that is, $P_{3}=\downarrow f\left(1_{P_{1}}\right)$. Then $f$ decomposes as $f=f_{3} \circ f_{1}$, where $f_{1}: P_{1} \rightarrow P_{3}$, defined by $f_{1}(x):=f(x)$, is a $\{0,1\}$ preserving isotone map and $f_{3}: P_{3} \rightarrow P_{2}$, defined by $x \mapsto x$, is a 0 -preserving injective isotone map.

Note that the embedding $f_{3}$ is necessarily 0 -separating. We can use Czédli [7] to represent $f_{1}$, while some ideas of Czédli [4] can be modified to represent $f_{3}$. Finally, the composite of these two representations is what we need in order to prove Theorem 1.2. Since Czédli [4] and [7] are long papers and it would take a lot 
of time of the reader to extract and appropriately modify ideas from them, we are going to outline these ideas by a concrete but sufficiently general example.

From now on, we denote the bottom element and the top element of $P_{i}$ by $0_{i}$ and $1_{i}$, respectively, while those of $L_{i}$ will be denoted by the boldface symbols $\mathbf{0}_{i}$ and $\mathbf{1}_{i}$. When no ambiguity threatens, we will often write $\left\langle P_{i}, \leq_{i}\right\rangle$ instead of $\left\langle P_{i}, \leq_{P_{i}}\right\rangle$. The least congruence and the largest congruence of a lattice $L$ will be denoted by $\Delta_{L}$ and $\nabla_{L}$, respectively. Let $L$ be an ordered set or a lattice. For $x, y \in L,\langle x, y\rangle$ is called an ordered pair of $L$ if $x \leq y$. If $y$ covers $x$, then $\langle x, y\rangle$ is an edge of $L$. Edges and prime intervals are essentially the same but edges are pairs of elements while prime intervals are two-element subsets. The set of ordered pairs of $L$ is denoted by Pairs $\leq(L)$. As opposed to the concept of intervals $[x, y]$, pairs and the notation $\langle x, y\rangle$ make it clear that $S \subseteq L$ implies that Pairs ${ }^{\leq}(S) \subseteq$ Pairs $\leq(L)$.

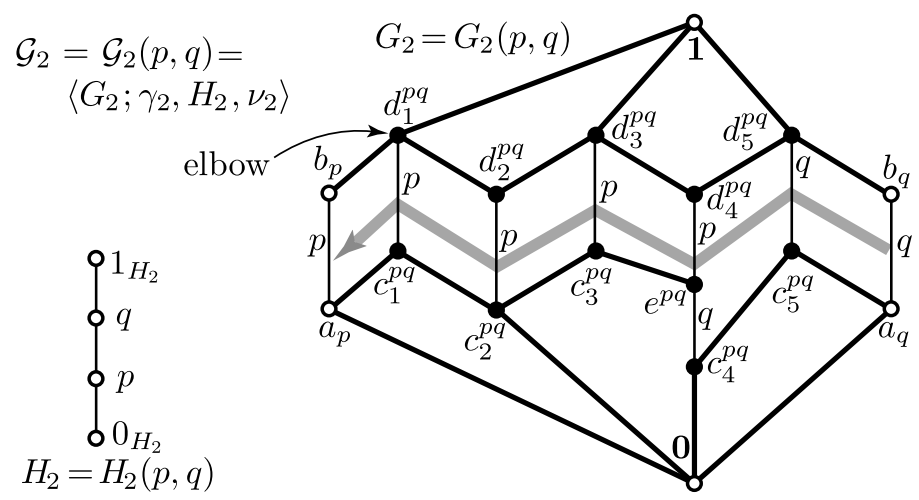

Figure 1. Our gadget $\mathcal{G}_{2}=\mathcal{G}_{2}(p, q)=\left\langle G_{2} ; \gamma_{2}, H_{2}, \nu_{2}\right\rangle$

3.2. Basic gadgets and their pictograms, the zigzag arrows. Our basic tool is the lattice $G_{2}=G_{2}(p, q)$ given on the right of Figure 1. This lattice is taken from Czédli [7], where it is denoted by $G_{2}^{\text {up }}(p, q)$, because [7] also uses its "down" variant. Some details of Figure 1 that are not needed at this stage will be explained later. Note that we can use $G_{2}$ and $\mathcal{G}_{2}$ with parameters other than $p$ and $q$, and we often drop the parameters if they are not relevant or they are clear from the context. The edges $\left\langle a_{q}, b_{q}\right\rangle$ and $\left\langle a_{p}, b_{p}\right\rangle$ are called the first edge and the target edge of $G_{2}(p, q)$, respectively. In order to make our figures less crowded, we will often denote $G_{2}$ by a grey zigzag arrow that is directed from its first edge to its target edge. We also say that the zigzag arrow goes from the first edge to the target edge. Sometimes we draw a double-lined zigzag arrow to indicate that besides a zigzag arrow some other elements (whose set will be denoted by $U_{p q}$ in our figures) are also added. We will explain later why we need double-lined zigzag arrows and we will define them exactly in (3.18); at present, it suffices to know that their role is the same as that of the "single-lined" zigzag arrows. Zigzag arrows without the adjective "double-lined" are always understood as single-lined ones. Observe that $\operatorname{con}\left(a_{p}, b_{p}\right)$ collapses only the $p$-labeled edges, so its non-singleton blocks are $\left\{a_{p}, b_{p}\right\},\left\{c_{1}^{p q}, d_{1}^{p q}\right\},\left\{c_{2}^{p q}, d_{2}^{p q}\right\},\left\{c_{3}^{p q}, d_{3}^{p q}\right\}$, and $\left\{e^{p q}, d_{4}^{p q}\right\}$. Similarly,

the non-singleton blocks of con $\left(a_{q}, b_{q}\right)$ are $\left\{a_{p}, b_{p}\right\},\left\{c_{1}^{p q}, d_{1}^{p q}\right\},\left\{c_{2}^{p q}, d_{2}^{p q}\right\}$, $\left\{c_{3}^{p q}, d_{3}^{p q}\right\},\left\{c_{4}^{p q}, e^{p q}, d_{4}^{p q}\right\},\left\{c_{5}^{p q}, d_{5}^{p q}\right\},\left\{c_{6}^{p q}, d_{6}^{p q}\right\}$, and $\left\{a_{q}, b_{q}\right\}$. 
The quotient lattices $G_{1}:=G_{2} / \operatorname{con}\left(a_{p}, b_{p}\right)$ and $G_{0}:=G_{2} / \operatorname{con}\left(a_{q}, b_{q}\right)$ and the corresponding "gadget structures" will be denoted by different kinds of grey zigzag arrow pictograms as Figures 2 and 3 show. These arrows will have no double-lined variants.

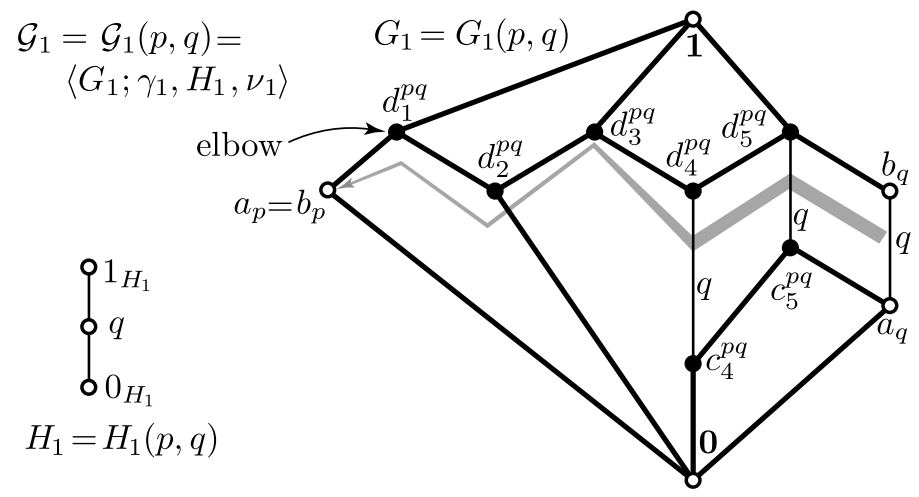

Figure 2. The quotient gadget $\mathcal{G}_{1}=\mathcal{G}_{1}(p, q)=\left\langle G_{1} ; \gamma_{1}, H_{1}, \nu_{1}\right\rangle$

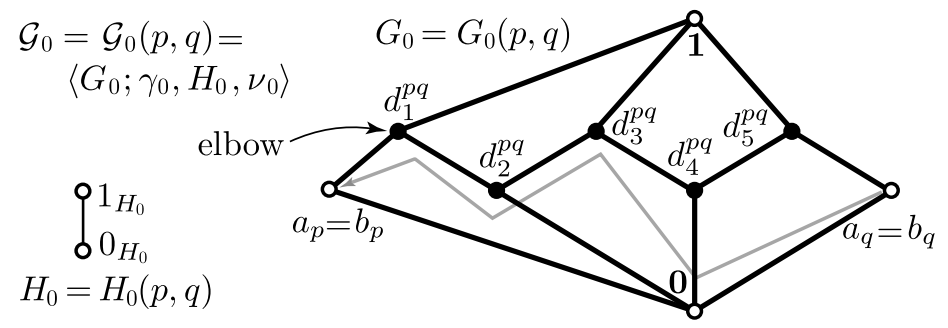

FigurE 3. The quotient gadget $\mathcal{G}_{0}=\mathcal{G}_{0}(p, q)=\left\langle G_{0} ; \gamma_{0}, H_{0}, \nu_{0}\right\rangle$

The zigzag arrow notation in Figure 1 and also in other figures is motivated by the way the congruences spread: $\operatorname{con}\left(a_{q}, b_{q}\right) \geq\left\langle a_{p}, b_{p}\right\rangle$, that is, $\operatorname{con}\left(a_{q}, b_{q}\right)$ collapses the $p$-colored edge $\left\langle a_{p}, b_{p}\right\rangle$. The lattice $G_{2}$ and its quotient lattices $G_{1}$ and $G_{0}$ will be referred to as our gadgets or zigzag arrows. Sometimes, $G_{1}$ and $G_{0}$ will be called "quotient zigzag arrows". Note that

$$
\begin{aligned}
& \operatorname{con}\left(a_{p}, b_{p}\right) \text { and } \operatorname{con}\left(a_{q}, b_{q}\right) \text { are the only } \\
& \text { nontrivial congruences of } G_{2}(p, q),
\end{aligned}
$$

whereby we will use only two kinds of quotient zigzag arrows. So, there are three different zigzag arrows, the "non-quotient" $\mathcal{G}_{2}$ and two quotient ones, $\mathcal{G}_{1}$ and $\mathcal{G}_{0}$. Now we premise our plans with them in our construction; this will hopefully help to enlighten the basic ideas, which will be detailed later. Note, however, that these plans will become more clear only in Subsection 3.3.

First, assume that we want to represent a single ordered set $\left\langle P ; \leq_{P}\right\rangle$ in the form $\langle\operatorname{Princ}(L) ; \subseteq\rangle$. Then we have to find a lattice $L$ and an order isomorphism $h:\left\langle P ; \leq_{P}\right\rangle \rightarrow\langle\operatorname{Princ}(L) ; \subseteq\rangle$. It will be clear from Subsection 3.3 soon that, for $p \in P$, we will let $h(p):=\operatorname{con}\left(a_{p}, b_{p}\right)$. Also, for $p<q$ in $P$, we will extend the set 
(in fact, the six-element sublattice) $\left\{0, a_{p}, b_{p}, a_{q}, b_{q}, 1\right\}$ to the zigzag arrow $G_{2}(p, q)$ of Figure 1; the reason is that the zigzag arrow

$$
G_{2}(p, q) \text { forces the inequality } \operatorname{con}\left(a_{p}, b_{p}\right) \leq \operatorname{con}\left(a_{q}, b_{q}\right)
$$

and this inequality is needed to guarantee that $h$ is isotone. We do not need quotient zigzag arrows for this purpose, because they force only that $\Delta_{L} \leq \operatorname{con}\left(a_{q}, b_{q}\right)$ and $\Delta_{L} \leq \Delta_{L}$, which automatically hold. However, even if they are superfluous at this stage, quotient zigzag arrows can be included, since they do not disturb the job of the "non-quotient" $G_{2}$ zigzag arrows.

Second, the situation becomes more involved when we want to represent the map $f$ from (3.1) (with the subscript 2 changed to 3 ) from $\left\langle P_{1} ; \leq_{1}\right\rangle$ to $\left\langle P_{3} ; \leq_{3}\right\rangle$. We will represent $\left\langle P_{1} ; \leq_{1}\right\rangle$ by an order isomorphism $h_{1}:\left\langle P_{1} ; \leq_{1}\right\rangle \rightarrow\left\langle\operatorname{Princ}\left(L_{1}\right) ; \subseteq\right\rangle$ without quotient zigzag arrows as explained in the previous paragraph. But then we will face the problem that for $0<_{1} p<_{1} q$ in $P_{1}$, it may happen that, say, $0_{3}=f_{1}(p)<_{3} f_{1}(q)$ in $P_{3}$. Since $f_{1}$ is intended to be represented as $\operatorname{Princ}\left(g_{1}\right)$, see (1.1) (but replace the subscript 2 by 3), it follows from (1.2) (after slight notational changes) that

$$
\begin{aligned}
\Delta_{L_{3}} & =h_{3}\left(0_{3}\right)=h_{3}\left(f_{1}(p)\right)=\operatorname{Princ}\left(g_{1}\right)\left(h_{1}(p)\right) \\
& =\operatorname{Princ}\left(g_{1}\right)\left(\operatorname{con}\left(a_{p}, b_{p}\right)\right)=\operatorname{con}\left(g_{1}\left(a_{p}\right), g_{1}\left(b_{p}\right)\right) .
\end{aligned}
$$

This means that $g_{1}$ collapses $a_{p}$ and $b_{p}$, that is, $\left\langle a_{p}, b_{p}\right\rangle \in \operatorname{Ker}\left(g_{1}\right)$. On the other hand, a calculation similar to (3.5) shows that $\left\langle a_{q}, b_{q}\right\rangle \notin \operatorname{Ker}\left(g_{1}\right)$. Hence, it follows from (3.3) that $g_{1}$ maps the $G_{2}(p, q)$ sublattice of $L_{1}$ onto a quotient zigzag arrow $G_{1}$. So even if $G_{1}$ would not be necessary to represent $\left\langle P_{3} ; \leq_{3}\right\rangle$ in itself, some copies of $G_{1}$ has to be included in $L_{3}$, because otherwise we could not define an appropriate lattice homomorphism $g_{1}: L_{1} \rightarrow L_{3}$. The motivation for using $G_{0}$ is similar but it has an additional feature. Namely, if $0_{3}=f_{1}(p)=f_{1}(q)$, then $\operatorname{Ker}\left(g_{1}\right)$ has to collapse each of the pairs $\left\langle a_{p}, b_{p}\right\rangle$ and $\left\langle a_{q}, b_{q}\right\rangle$, but it cannot collapse a pair that is not collapsed by the congruence of $G_{2}(p, q)$ described in (3.2), because otherwise $\left\langle 0_{L_{1}}, 1_{L_{1}}\right\rangle$ would belong to $\operatorname{Ker}\left(g_{1}\right)$ and $\operatorname{Ker}\left(g_{1}\right)$ would collapse the whole lattice $L_{1}$, so the range of $\operatorname{Princ}\left(g_{1}\right)$ would be the singleton set $\left\{\Delta_{L_{3}}\right\}$, which is clearly not the case in general. Combining this with (3.3), it follows that $g_{1}$ has to map $G_{2}(p, q)$ to a copy of $G_{0}$, provided that $0_{3}=f_{1}(p)=f_{1}(q)$.

Finally, the quotient zigzag arrows that are necessarily included in $L_{3}$ will not disturb us to extend $L_{3}$ to a lattice $L_{2}$ in a way similar to the one used in Czédli [4].

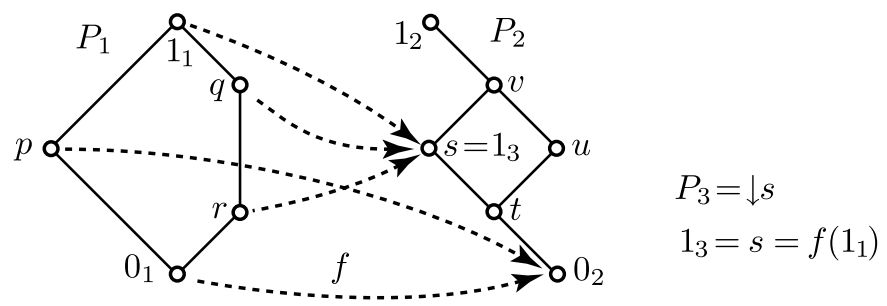

Figure 4. An example 


\subsection{Describing the construction with an example.}

Example 3.1. Let $P_{1}=\left\{0_{1}, p, q, r, 1_{1}\right\}$ and $P_{2}=\left\{0_{2}, s, t, u, v, 1_{2}\right\}$ be the ordered sets given in Figure 4, and let $f: P_{1} \rightarrow P_{2}$ be the isotone map indicated by dashed arrows in the figure.

Figure 5 shows how we represent $P_{1}$ as $\operatorname{Princ}\left(L_{1}\right)$. We start with the eight element simple lattice $M_{3,3}$; in Figure $5, M_{3,3}$ is the sublattice of $L_{1}$ formed by the pentagon-shaped elements. One of the edges of $M_{3,3}$ that is disjoint from $\{0,1\}$ is denoted by $\left\langle a_{1_{1}}, b_{1_{1}}\right\rangle$; this edge and all thick edges in the figure are colored by $1_{1} \in P_{1}$. In the next step, we add the dark-grey-filled large elements. That is, for every $x \in P_{1} \backslash\left\{0_{1}, 1_{1}\right\}$, we add the thin edge $\left\langle a_{x}, b_{x}\right\rangle$. We often call this edge a basic edge. Our goal is that the principal congruence $\operatorname{con}\left(a_{x}, b_{x}\right)$ should represent $x \in P_{1}$. That is, the map

$$
h_{1}: P_{1} \rightarrow \operatorname{Princ}\left(L_{1}\right), \quad \text { defined by } \quad x \mapsto \operatorname{con}\left(a_{x}, b_{x}\right),
$$

should be an order isomorphism. At present, we are far from this goal since the principal congruences $\operatorname{con}\left(a_{x}, b_{x}\right)$, for $x \in P_{1} \backslash\left\{0_{1}, 1_{1}\right\}$, form an antichain. Therefore, we add several copies of our gadget $G_{2}$ in order to force the comparability of $\operatorname{con}\left(a_{x}, b_{x}\right)$ and $\operatorname{con}\left(a_{y}, b_{y}\right)$ whenever $x, y \in L \backslash\left\{0_{1}, 1_{1}\right\}$ are comparable. We can add a gadget going from the basic edge $\left\langle a_{y}, b_{y}\right\rangle$ to the basic edge $\left\langle a_{x}, b_{x}\right\rangle$ for every $\langle x, y\rangle \in$ Pairs $\leq\left(P_{1} \backslash\left\{0_{1}, 1_{1}\right\}\right)$, but it is often sufficient to add less gadgets because of transitivity. Note that the gadget added to $\left\langle p, 1_{1}\right\rangle$, indicated only by a (thick grey) zigzag arrow, is superfluous in Figure 5; it is in the figure to exemplify later how to deal with the $f$-preimages of $0_{3}=0_{2}$.

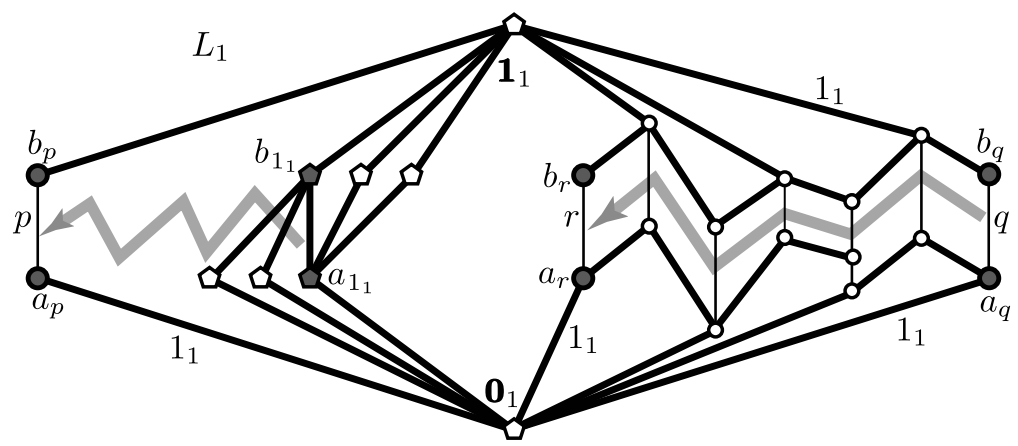

FiguRe 5. $P_{1} \cong \operatorname{Princ}\left(L_{1}\right)$

As Figure 6 shows, the representation of $P_{3}=\downarrow s$ as $\operatorname{Princ}\left(L_{3}\right)$ is similar but we need some new features: $L_{3}$ has an extra element $a_{0(p)}=b_{0(p)}$, it has two $s$-colored thin basic edges, and there are gadgets, in both directions, between the $s$-colored basic edges. Also, to guarantee that the $s$-colored basic edges generate $\nabla_{L_{3}}$, a zigzag arrow goes from the basic edge $\left\langle a_{s(q)}, b_{s(q)}\right\rangle$ to the edge $\left\langle a_{1_{3}}, b_{1_{3}}\right\rangle$. Note that some edges ending at $\mathbf{1}_{3}$ or starting from $\mathbf{0}_{3}$ need not indicate coverings in Figure 6; for example, since the $t$-colored basic edge $\left\langle a_{t}, b_{t}\right\rangle$ is the target edge of a zigzag arrow, we have only that $b_{t}<\mathbf{1}_{3}$ but $b_{t} \nprec \mathbf{1}_{3}$. This will not cause any problem in what follows, and

$$
h_{3}: P_{3} \rightarrow \operatorname{Princ}\left(L_{3}\right), \quad \text { defined by } \quad x \mapsto \operatorname{con}\left(a_{x}, b_{x}\right),
$$


is an order isomorphism.

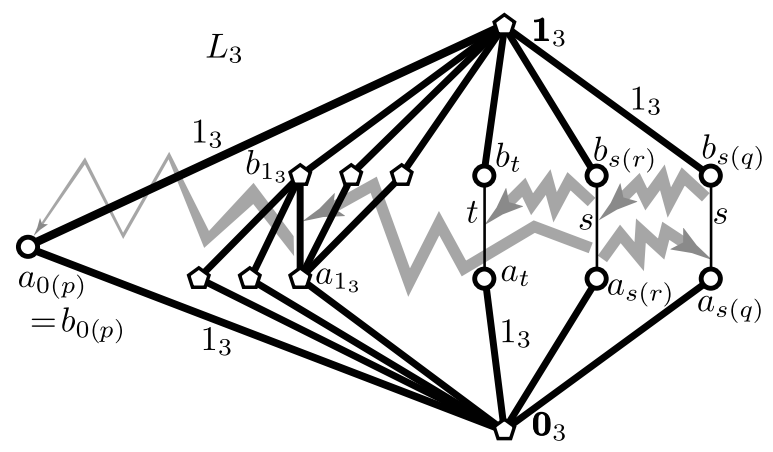

Figure $6 . P_{3} \cong \operatorname{Princ}\left(L_{3}\right)$

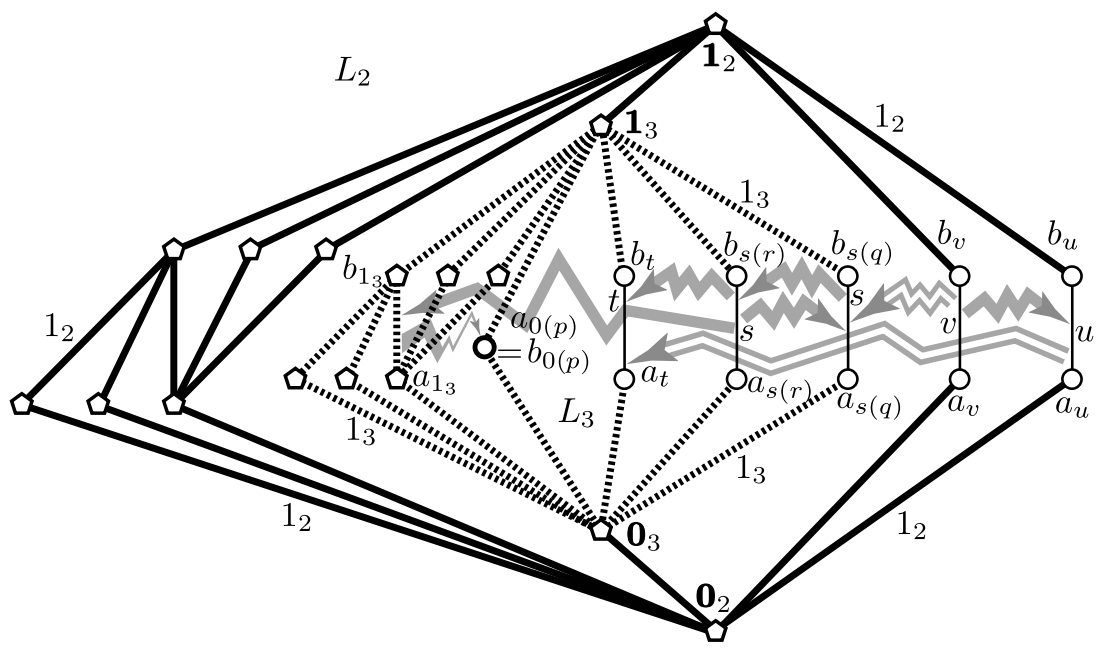

Figure $7 . P_{2} \cong \operatorname{Princ}\left(L_{2}\right)$

The required homomorphism $g_{1}: L_{1} \rightarrow L_{3}$ is defined as follows. It maps the $M_{3,3}$ sublattice, which is the collection of the pentagon-shaped elements of $L_{1}$, onto the $M_{3,3}$ sublattice of $L_{3}$ such that $g_{1}\left(a_{1_{1}}\right)=a_{1_{3}}$ and $g_{1}\left(b_{1_{1}}\right)=b_{1_{3}}$. Motivated by $f(p)=0_{3}, f$ maps both $a_{p}$ and $b_{p}$ to $a_{0(p)}=b_{0(p)}$. The element $s \in P_{3}$ has two $f$-preimages in $P_{1} \backslash\left\{1_{1}\right\}$; this explains that $L_{3}$ has two $s$-colored thin basic edges. The pairs $\left\langle a_{q}, b_{q}\right\rangle$ and $\left\langle a_{r}, b_{r}\right\rangle$ are mapped to the pairs $\left\langle a_{s(q)}, b_{s(q)}\right\rangle$ and $\left\langle a_{s(r)}, b_{s(r)}\right\rangle$, respectively. The left and the right grey zigzag arrows (representing copies of $G_{2}$ ) of $L_{1}$ are mapped to the leftmost grey zigzag arrow and the rightmost upper zigzag arrow, respectively. Since $G_{1}$, the leftmost zigzag arrow in Figure 6 , is a homomorphic image of $G_{2}$, it is easy to see that the map $g_{1}$ we have just defined is a lattice homomorphism. It is straightforward to see, at least for Example 3.1, that 
see Definition 1.1. Note that since $\operatorname{con}\left(a_{q}, a_{r}\right)=\nabla_{L_{1}}$, it follows from (1.1) and $f=\operatorname{Princ}(g)$ that $g\left(a_{q}\right) \neq g\left(a_{r}\right)$; this explains why we need two $s$-colored basic edges in $L_{3}$. So far, we have not used any double-lined zigzag arrow.

As the last step of the construction, we extend $L_{3}$ to a lattice $L_{2}$ as shown in Figure 7 . In this figure, $L_{3}$ is the interval $\left[\mathbf{0}_{3}, \mathbf{1}_{3}\right]$, and

each of the thick dotted edges of $L_{3}$ generates a congruence that corresponds to $1_{3} \in P_{3}$.

In order to take care of the comparability $u \leq v$, there is a new (single-lined) zigzag arrow in $L_{2}$ with first edge $\left\langle a_{v}, b_{v}\right\rangle$ and target edge $\left\langle a_{u}, b_{u}\right\rangle$. ("New" means that it is not in $L_{3}$.) This is possible because at the beginning, as previously, the top element $b_{u}$ of the target edge is a coatom in $L_{2}$, so the new zigzag arrow lies in $L_{2}$ basically in the same way as the zigzag arrows lied in $L_{1}$ and $L_{3}$. However, we use double-lined zigzag arrows in $L_{2}$ to take care of the comparabilities $s \leq v$ and $t \leq u$; we will explain later in (3.18) what these double-lined zigzag arrows are, and we will point out why a single-lined zigzag arrow cannot work if there is an edge $\mathfrak{r}$ in the filter generated by the top of its target edge such that $\operatorname{con}(\mathfrak{r}) \neq \nabla_{L_{2}}$.

Now that the new arrows, single-lined and double-lined, take care of each of the comparabilities $u \leq v, s \leq v, t \leq s$, and $t \leq u$, it follows that

$$
h_{2}: P_{2} \rightarrow \operatorname{Princ}\left(L_{2}\right), \quad \text { defined by } \quad x \mapsto \operatorname{con}\left(a_{x}, b_{x}\right),
$$

is an order isomorphism. Let $g_{3}$ be the natural embedding

$$
g_{3}: L_{3} \rightarrow L_{2} \text {, defined by } x \mapsto x .
$$

It is straightforward to see, at least for Example 3.1, that

$$
g_{3} \text { represents } f_{3} \text { by means of principal congruences. }
$$

Let $g=g_{3} \circ g_{1}$; it is a lattice homomorphism from $L_{1}$ to $L_{2}$. We know from Czédli $[6,7]$ and it is easy to see that Princ is a functor, whereby

$$
\operatorname{Princ}\left(g_{3}\right) \circ \operatorname{Princ}\left(g_{1}\right)=\operatorname{Princ}\left(g_{3} \circ g_{1}\right)=\operatorname{Princ}(g) \text {. }
$$

For $i \in\{1,3\}$, let $h_{i}: P_{i} \rightarrow \operatorname{Princ}\left(L_{i}\right)$ denote the order isomorphism defined by $x \mapsto \operatorname{con}\left(a_{x}, b_{x}\right)$; see (3.6), (3.7), and (3.10). By (1.2), (3.8) and (3.12) mean that

$$
f_{1}=h_{3}^{-1} \circ \operatorname{Princ}\left(g_{1}\right) \circ h_{1} \quad \text { and } \quad f_{3}=h_{2}^{-1} \circ \operatorname{Princ}\left(g_{3}\right) \circ h_{3} .
$$

Combining (3.13) and (3.14), we obtain that

$$
\begin{aligned}
f & =f_{3} \circ f_{1}=\left(h_{2}^{-1} \circ \operatorname{Princ}\left(g_{3}\right) \circ h_{3}\right) \circ\left(h_{3}^{-1} \circ \operatorname{Princ}\left(g_{1}\right) \circ h_{1}\right) \\
& =h_{2}^{-1} \circ \operatorname{Princ}\left(g_{3}\right) \circ \operatorname{Princ}\left(g_{1}\right) \circ h_{1}=h_{2}^{-1} \circ \operatorname{Princ}(g) \circ h_{1} .
\end{aligned}
$$

Hence, $g$ is representable by principal congruences of lattices of lengths 5 and 7 .

3.4. The construction for the general case. The construction for the general case is almost the same as that for Example 3.1. Hence, it suffices to point out the differences. The construction of $L_{1}$ is essentially the same as in the example.

For each $p \in f^{-1}\left(0_{2}\right), L_{3}$ has to contain an element $a_{0(p)}=b_{0(p)}$ that is an atom and also a coatom in $L_{2}$. Remember that $0_{2}=0_{3}$. Of course, $g_{1}$ maps the elements $a_{p}$ and $b_{p}$ of $L_{1}$ to the element $a_{0(p)}=b_{0(p)} \in L_{3}$. Note that for $p, p^{\prime} \in f^{-1}\left(0_{2}\right)$, if $p \neq p^{\prime}$, then $a_{0(p)} \neq a_{0\left(p^{\prime}\right)}$. For each $s \in f\left(P_{1}\right) \backslash\left\{0_{3}\right\}$, we need as many $s$ colored thin basic edges in $L_{3}$ as the size $\left|f^{-1}(s) \backslash\left\{1_{1}\right\}\right|$ of $f^{-1}(s) \backslash\left\{1_{1}\right\}$. So if $f^{-1}(s) \backslash\left\{1_{1}\right\}=\{q, r, \ldots\}$, then we include the $s$-colored basic edges $\left\langle a_{s(q)}, b_{s(q)}\right\rangle$, $\left\langle a_{s(r)}, b_{s(r)}\right\rangle, \ldots$ in $L_{3}$. In order to guarantee that every $s$-colored edge generates 
the same congruence of $L_{3}$, we let a zigzag arrow go between any two $s$-colored basic edges in both directions. (Note that it often suffices to use fewer zigzag arrows; we only need that the "reflexive and transitive closure of the zigzag arrows" is the full relation on the set of $s$-colored edges of $L_{3}$.) So far, we have seen what $L_{3}$ is and we have defined the action of $g_{1}$ for the $M_{3,3}$ sublattice of $L_{1}$ and for the thin basic edges of $L_{1}$.

In the next step, we extend the action of $g_{1}$ to the gadgets. For each gadget, that is, for each zigzag arrow $Z \cong G_{2}$ of $L_{1}$, we do the following. Let $\left\langle a_{h}, b_{h}\right\rangle$ and $\left\langle a_{w}, b_{w}\right\rangle$ be the target edge and the first edge of $Z$, respectively, and observe that since $Z$ is included in $L_{1}$, we have that $h \leq_{1} w$ in $P_{1}$. Thus, $f(h) \leq_{3} f(w)$ in $P_{3}$ since $f$ is isotone, and there are three cases to consider.

First, if $f$ maps none of $h$ and $w$ to $0_{3}$, then $\left\langle g_{1}\left(a_{h}\right), g_{1}\left(b_{h}\right)\right\rangle=\left\langle a_{f(h)}, b_{f(h)}\right\rangle$ and $\left\langle g_{1}\left(a_{w}\right), g_{1}\left(b_{w}\right)\right\rangle=\left\langle a_{f(w)}, b_{f(w)}\right\rangle$ are basic edges of $L_{3}$ and $L_{3}$ contains a zigzag arrow $Z^{\prime} \cong G_{2}$ from $\left\langle g_{1}\left(a_{w}\right), g_{1}\left(b_{w}\right)\right\rangle$ to $\left\langle g_{1}\left(a_{h}\right), g_{1}\left(b_{h}\right)\right\rangle$ by the construction of $L_{3}$. In this case, $g_{1}$ restricted to $Z$ will be an isomorphism from $Z$ to $Z^{\prime}$.

Second, if $f(h)=0_{3} \neq f(w)$, then we modify $L_{3}$ by adding a quotient zigzag arrow $G_{1}$ that goes from $\left\langle g_{1}\left(a_{w}\right), g_{1}\left(b_{w}\right)\right\rangle=\left\langle a_{f(w)}, b_{f(w)}\right\rangle$ to $\left\langle g_{1}\left(a_{h}\right), g_{1}\left(b_{h}\right)\right\rangle=$ $\left\langle a_{0(h)}, b_{0(h)}\right\rangle$. Observe that $a_{0(h)}=b_{0(h)}$ and so $\operatorname{con}\left(a_{0(h)}, b_{0(h)}\right)=\Delta_{L_{3}}$. Hence, the new zigzag arrow does not spoil the construction of $L_{3}$ since its only effect is to force the inequality $\Delta_{L_{3}} \leq \operatorname{con}\left(a_{f(w)}, b_{f(w)}\right)$, which holds automatically.

Third, if $f(h)=0_{3}=f(w)$, then we add a quotient zigzag arrow $G_{0}$ going from the "degenerate" (singleton) edge $\left\langle g_{1}\left(a_{w}\right), g_{1}\left(b_{w}\right)\right\rangle=\left\langle a_{0(w)}, b_{0(w)}\right\rangle$ to the degenerate edge $\left\langle g_{1}\left(a_{h}\right), g_{1}\left(b_{h}\right)\right\rangle=\left\langle a_{0(h)}, b_{0(h)}\right\rangle$; this does not spoil anything.

Finally, we extend $L_{3}$ to $L_{2}$ and we define $g_{3}$ in the same way as in Example 3.1. Since $P_{3}$ is a (principal) order ideal in $P_{2}$, there are only two sorts of comparabilities $u \leq v$ in $P_{2}$ that we still have to force, namely,

$$
\begin{aligned}
& \text { either } u, v \in P_{2} \backslash P_{3} \text { and } u \leq v, \\
& \text { or } u \in P_{3} \text { and } v \in P_{2} \backslash P_{3} \text { and } u \leq v \text {. }
\end{aligned}
$$

In case of the first alternative mentioned in (3.15), every edge $\langle x, y\rangle$ with $x \geq b_{u}$ is a thick and solid edge and generates the largest congruence; as a consequence to be clarified later, we use a (single-lined) zigzag arrow in this case. In the second case, we use a double-lined zigzag arrow; we are going to point out a few lines later why.

Note at this point that

a zigzag arrow "arrives" at its target edge from above and "departs" from its first edge upwards;

see our figures. Therefore, as it will be explained later (with reference to the present paragraph), it needs a special attention whether all the edges above the target edge are thick and solid or not, but it is irrelevant whether the same holds below the target edge and below the first edge. As opposed to Czédli [4], now since all edges above $b_{v}$ are thick and solid for both alternatives given in (3.15), the first edges of the new single-lined or double-lined zigzag arrows will need no special care.

For $p \in P_{3} \backslash\left\{0_{3}\right\}$, let

$$
U_{p}:=\left[b_{p}, \mathbf{1}_{3}\right] \text {, which is a filter in } L_{3} \text { and an interval in } L_{2} .
$$

The element $d_{1}^{p q}$ in Figure 1 will be called the elbow of $G_{2}(p, q)$. By the construction of $L_{3}, U_{p}$ consists of $b_{p}, \mathbf{1}_{3}$, and the elbows of the zigzag arrows in $L_{3}$ with target edge $\left\langle a_{p}, b_{p}\right\rangle$. (It may happen that there is no such elbow; then $\left|U_{p}\right|=2$. If $\left|U_{p}\right|>2$, then it is a modular lattice of length 2.) For $p \in P_{3} \backslash\left\{0_{3}\right\}$ and $q \in P_{2} \backslash P_{3}$, inserting 
a double-lined zigzag arrow with first edge $\left\langle a_{q}, b_{q}\right\rangle$ and target edge $\left\langle a_{p}, b_{p}\right\rangle$ means that

first we insert a (single-lined) zigzag arrow, and then we add a new interval $U_{p q}$ isomorphic to $U_{p}$ such that $\left[b_{p}, 1_{U_{p q}}\right]$ is isomorphic to the direct product of $U_{p}$ and the two-element chain $\{0,1\}$ such that $U_{p}$ corresponds to $U_{p} \times\{0\}$ in $\left[b_{p}, 1_{U_{p q}}\right]$;

see Figures 8 and 9 for illustration. In both figures, $U_{p}$ is the lowest grey-filled interval and it consists of the black-filled circles and the black-filled pentagon, while the interval $U_{p q}$ is also grey-filled and it consists of the grey-filled square elements.

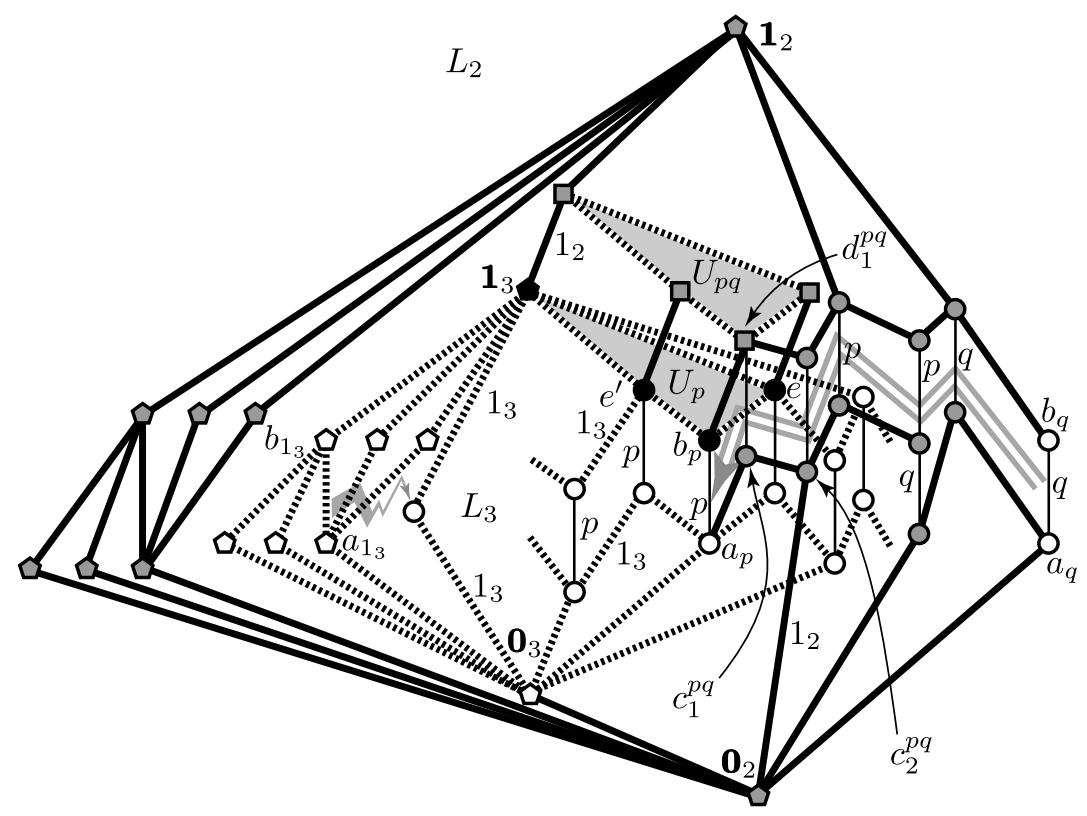

FiguRE $8 . U_{p}=\{$ black-filled elements $\}$, a double-lined zigzag arrow from $\left\langle a_{q}, b_{q}\right\rangle$ to $\left\langle a_{p}, b_{p}\right\rangle$, and a part of $L_{2}$ (not for Example 3.1)

Note that (3.9) is still valid; in fact, our intention to preserve its validity explains why we cannot use (single-lined) zigzag arrows instead of double-lines ones here. Namely, continuing the paragraph containing (3.16), remember that the bottom element of $U_{p q}$ is $d_{1}^{p q}$, the elbow element of the zigzag arrow $G_{2}(p, q)$. Assume that we delete $U_{p q} \backslash\left\{d_{1}^{p q}\right\}$ from Figure 8 or from Figure 9 . Then the elbow $d_{1}^{p q}$ becomes a coatom and $\left\langle d_{1}^{p q}, \mathbf{1}_{2}\right\rangle$ becomes a solid thick edge, that is, it generates the largest congruence of $L_{2}$ and so it corresponds to the top $1_{2}$ of $P_{2}$. However, then the dotted thick edge $\left\langle b_{p}, e^{\prime}\right\rangle$ and the solid thick edge $\left\langle d_{1}^{p q}, \mathbf{1}_{2}\right\rangle$ become transposed, and so they generate the same congruence, which violates (3.9). Furthermore, it remains true that any two thick dotted edges generate the same congruence, and it turns out that no congruence of $L_{2}$ corresponds to $1_{3} \in P_{3}$; this is what we surely have to avoid. It will turn out that the usage of double-lined zigzag arrows is sufficient to keep the validity of (3.9), and our $L_{2}$ does the job. 
As opposed to the top element $b_{p}$ of the basic edge associated with $p \in P_{3} \backslash\left\{0_{3}\right\}$, its bottom element $a_{p}$ does not cause a similar difficulty. So, as opposed to $U_{p q}$ inserted above the single-lined part $Z$ of the double-lined zigzag arrow from $\left\langle a_{q}, b_{q}\right\rangle$ to $\left\langle a_{p}, b_{p}\right\rangle$, we do not have to add extra elements below $Z$. In order to give a first impression why this is so, note that the ideal $\downarrow c_{1}^{p q}=\left\{\mathbf{0}_{2}, \mathbf{0}_{3}, a_{p}, c_{2}^{p q}, c_{1}^{p q}\right\}$ is a sublattice isomorphic to $N_{5}$; see Figures 8 and 9 . Obviously, the congruence $\operatorname{con}_{\downarrow c_{1}^{p q}}\left(\mathbf{0}_{3}, a_{p}\right)$ generated by the dotted thick edge of this sublattice does not collapse any solid thick edge in this sublattice; much less obviously, the same will turn out to hold for $\operatorname{con}_{L_{2}}\left(\mathbf{0}_{3}, a_{p}\right)$ in the whole lattice $L_{2}$.

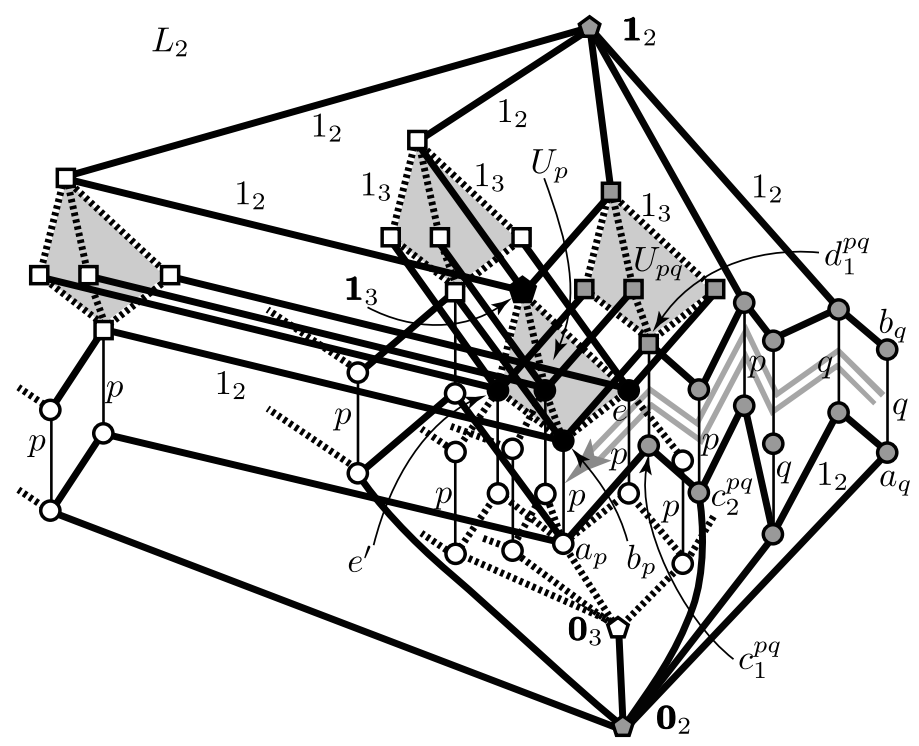

FiguRE 9. Adding the third double-lined zigzag arrow with target edge $\left\langle a_{p}, b_{p}\right\rangle$ and a part of $L_{2}$ (not for Example 3.1)

\section{Proving that our CONStRuction WORKS}

In this section, we are going to prove that our construction has the properties stated in Section 3; this will imply Theorem 1.2. A direct proof given in a selfcontained way with all details would result in an extremely long paper, which we want to avoid. Therefore, we organize the proof so that it relies on very similar considerations, even if this makes it necessary to reference some long proofs in addition to some statements from earlier papers. First, we claim that (3.8) holds in general, not only for our example.

Lemma 4.1. The lattice homomorphism $g_{1}: L_{1} \rightarrow L_{3}$ constructed in the previous section represents $f_{1}$ by means of principal congruences.

Proof. The proof of the main result in Czédli [7] yields this lemma as the particular case where only one 0-preserving isotone map between two bounded ordered sets has to be represented. In order to make this observation clear, note that the main difference between the present construction and that in [7] is the following. Here we use only one gadget $G_{2}$ to force an inequality mentioned in (3.4). The same 
inequality in [7] is forced twice; once with $G_{2}$ and once with the dual of $G_{2}$. The reason is that [7] constructs selfdual lattices; we do not pursue a similar target, because that would make the rest of this section much more complicated.

Clearly, the above-mentioned "main difference" does not threaten the validity of Lemma 4.1, because of two obvious reasons. First, it suffices to force an inequality from (3.4) only once. Second, it is even safer to force it only once, because otherwise it is more difficult to show that a different additional forcing does not force nondesired inequalities.

4.1. Quasi-colored lattices. In this subsection, we recall a concept, which has been useful in Czédli $[1,4]$; it will be used while proving that the lattice homomorphism (in fact, embedding) $g_{3}: L_{3} \rightarrow L_{2}$ represents $f_{3}$. A quasiordered set, also known as a preordered set, is a structure $\langle H ; \nu\rangle$ where $H \neq \varnothing$ is a set and $\nu \subseteq H^{2}$ is a reflexive and transitive relation on $H$. We often use the notation $x \leq_{\nu} y$ instead of $\langle x, y\rangle \in \nu$. For $X \subseteq H^{2}$, the least quasiorder on $H$ that includes $X$ is denoted by $\operatorname{quo}(X)$. We write quo $(x, y)$ instead of quo $(\{\langle x, y\rangle\})$. The advantage of using quasiorders over partial orderings is that quo $(X)$ always exists. This fact is extremely useful in constructions where we modify a quasiorder by adding new pairs to it. Since antisymmetry is inherited by smaller relations, it follows that

If $\left\{\nu_{i}: i \in I\right\}$ is a set of quasiorders on $H$ such that there is a partial order $\widehat{\nu}$ with $\nu_{i} \subseteq \widehat{\nu}$ for all $i \in I$, then all the $\nu_{i}$ and quo $\left(\bigcup_{i \in K} \nu_{i}\right)$ are also partial orders on $H$.

Following Czédli $[1,4]$, a quasi-colored lattice is a structure $\mathcal{L}=\langle L ; \gamma, H, \nu\rangle$ where $L$ is a lattice, $\langle H ; \nu\rangle$ is a quasiordered set, $\gamma:$ Pairs $^{\leq}(L) \rightarrow H$ is a surjective map, and for all $\left\langle u_{1}, v_{1}\right\rangle,\left\langle u_{2}, v_{2}\right\rangle \in$ Pairs $^{\leq}(L)$,

(C1) if $\gamma\left(\left\langle u_{1}, v_{1}\right\rangle\right) \leq{ }_{\nu} \gamma\left(\left\langle u_{2}, v_{2}\right\rangle\right)$, then $\operatorname{con}\left(u_{1}, v_{1}\right) \leq \operatorname{con}\left(u_{2}, v_{2}\right)$;

(C2) if $\operatorname{con}\left(u_{1}, v_{1}\right) \leq \operatorname{con}\left(u_{2}, v_{2}\right)$, then $\gamma\left(\left\langle u_{1}, v_{1}\right\rangle\right) \leq_{\nu} \gamma\left(\left\langle u_{2}, v_{2}\right\rangle\right)$.

For example, $\mathcal{G}_{2}=\mathcal{G}_{2}(p, q)=\left\langle G_{2} ; \gamma_{2}, H_{2}, \nu_{2}\right\rangle$ in Figure 1 is a quasi-colored lattice. In this figure, all the thick edges are $1=1_{H_{2}}$-colored. Furthermore, if $x<y$, then $\gamma_{2}(\langle x, y\rangle)$ is the join of the colors of the edges in $[x, y]$ in the figure; the join is taken in the chain $\left\langle H_{2}, \nu_{2}\right\rangle$. This quasi-colored lattice as well as the quasi-colored lattices in Figures 2 and 3 are taken from Czédli [7]. If $\langle H ; \nu\rangle$ happens to be an ordered set, then $\mathcal{L}$ above is a colored lattice. As a consequence of (4.1),

all the quasi-colored lattices we are going construct in this paper will be colored lattices.

The importance of (4.2) lies in the fact that we know from Czédli [7] or, less explicitly, from [4, Lemma 2.1] that for every colored lattice $\mathcal{L}=\langle L ; \gamma, H, \nu\rangle$, the map

$$
h: H \rightarrow \operatorname{Princ}(L), \quad \text { defined by } \quad p \mapsto \operatorname{con}(\text { a } p \text {-colored edge), }
$$

is an order isomorphism. Note that $h$ above is well defined, since (C1) implies that no matter which $p$-colored edge is considered in (4.3).

4.2. Completing the proof with [4]. If $P_{i}$ is a singleton and $P_{i} \cong \operatorname{Princ}\left(L_{i}\right)$, then $L_{i}$ is necessarily the 1-element lattice, which cannot be obtained by our construction. However, if $\left|P_{i}\right|=1$ for some $i \in\{1,2\}$, then Theorem 1.2 follows from Grätzer [14], which represents $P_{3-i}$ as $\operatorname{Princ}\left(L_{3-i}\right)$ with $L_{3-i}$ of length at most 5 . 
Hence, in what follows, we assume that none of $P_{1}$ and $P_{2}$ is a singleton. In order to complete the proof of Theorem 1.2, we need to show only the following lemma.

Lemma 4.2. (3.12) holds in general, that is, $g_{3}$ represents $f_{3}$ by means of principal congruences.

We present two proofs, which are close to each other; the first one is less detailed and it is recommended only to those who are familiar not only with the statements but also with the proofs given in Czédli [4].

First proof of Lemma 4.2. The lemma follows from straightforward modifications of the method used in Czédli [4]. While extracting the proof of Lemma 4.2 from [4], the following three facts have to be taken into account.

First, since [4] deals with lattices without bottom and top elements and infinitely many lattice homomorphisms corresponding to our $g_{3}$ are constructed for an increasing sequence of ordered sets, [4] uses wider gadgets. Analyzing the proof of [4], one can see that $\mathcal{G}_{2}$ also works in the present particular case. There is another possibility: after constructing $L_{1}, L_{3}$, and $g_{1}: L_{1} \rightarrow L_{3}$, we could change our zigzag arrow $\mathcal{G}_{2}$ to the gadget used in [4]; see [4, Figure 2]; this would change the definition of $L_{2}$ and $g_{3}$ but Lemma 4.2 would remain valid.

Second, the role of our $U_{p q}$ corresponds to that of $U_{p}^{q}$ in [4] and some similar convex sublattices also occur there; see the grey-filled sublattices in [4, Figure 8]. The purpose of these convex sublattices is the generalization of (3.9), which cannot be achieved without some auxiliary subsets; see the last two paragraphs of Section 3 here. Here the situation is easier, because some of the grey-filled convex sublattices of [4, Figure 8] are singletons here and, as it was pointed out in the last paragraph of Section 3, some others cause no problem.

Third, whenever we add a gadget together with new grey-filled convex sublattices in [4], the length of the lattice can increase; this is not a problem there since at the end of the transfinite process, a lattice of infinite length is constructed. As opposed to [4], when we add a new double-lined zigzag arrow, then the new interval $U_{p q}$ is never put above an earlier $U_{p^{\prime} q^{\prime}}$. Hence, the length of the lattice does not increase when we add the second, third, etc. double-lined zigzag arrows. This is why we use $U_{p q}$ rather than the set $U_{p}^{q}$ from [4]. However, this modification does not change the argument of [4] significantly.

Taking the above-mentioned three facts into account, the method of [4] proves Lemma 4.2 .

Second proof of Lemma 4.2. It follows from the construction of $L_{3}$ that we have a coloring $\gamma^{\prime}:\left\langle H_{3}, \leq_{3}\right\rangle \rightarrow$ Pairs ${ }^{\leq}\left(L_{3}\right)$. Namely, for a prime interval $\mathfrak{p}, \gamma^{\prime}(\mathfrak{p})$ is the label of the edge $\mathfrak{p}$. If an edge is not labeled because of space considerations, then $\gamma^{\prime}(\mathfrak{p})$ is defined by the following two rules: the thick solid edges of $L_{3}$ are labeled by $1_{3}$, and if $\mathfrak{r}$ and $\mathfrak{r}^{\prime}$ are transposed edges in the same gadget $\mathcal{G}_{2}$, then $\gamma^{\prime}\left(\mathfrak{r}^{\prime}\right)=\gamma^{\prime}(\mathfrak{r})$. Of course, $\gamma^{\prime}(\langle x, x\rangle)=0_{3}=0_{2}$. Furthermore, if $\mathfrak{r}=\langle x, y\rangle$ such that $x<y$ but $y$ does not cover $x$, then take a maximal chain $x=z_{0} \prec z_{1} \prec \cdots \prec z_{n}=y$ in the interval $[x, y]$ and let

$$
\gamma^{\prime}(\mathfrak{r})=\gamma^{\prime}(\langle x, y\rangle):=\bigvee_{i=1}^{n} \gamma^{\prime}\left(\left\langle z_{i-1}, z_{i}\right\rangle\right),
$$

where the join is taken in $\left\langle P_{3}, \leq_{3}\right\rangle$. Of course, $\left\langle P_{3}, \leq_{3}\right\rangle$ is not a lattice and joins in it do not make sense in general. However, the set on the right of (4.4) is a finite 
chain or it contains $1_{3}$, the top element of $P_{3}$, whereby the join in (4.4) always makes sense; compare this with Lemma [4, Chain Lemma 4.6]. Furthermore, it is easy to see from the structure of $L_{3}$ that the join above does not depend on the choice of the maximal chain in $[x, y]$. Compare (4.4) also with the well-known fact that in a lattice of finite length, if $x=z_{0} \prec z_{1} \prec \cdots \prec z_{n}=y$ is a maximal chain in an interval $[x, y]$, then

$$
\operatorname{con}(x, y)=\bigvee_{i=1}^{n} \operatorname{con}\left(z_{i-1}, z_{i}\right)=\bigvee\left\{\operatorname{con}\left(\mathfrak{r}^{\prime}\right): x \leq 0_{\mathfrak{r}^{\prime}} \prec 1_{\mathfrak{r}^{\prime}} \leq y\right\} .
$$

Since $\left\langle\operatorname{Princ}\left(L_{3}\right) ; \subseteq\right\rangle$ represents $\left\langle P_{3}, \leq_{3}\right\rangle$, it is straightforward to derive from its construction that $\gamma^{\prime}$ is a quasi-coloring; in fact, it is a coloring.

The construction of $L_{2}$ begins with adding some new edges to $L_{3}$ that are either labeled or their thick solid style means that their labels are $1_{2}$; the earlier $1_{3}$-labeled edges become dotted and thick; see Figures 7-9 without the zigzag arrows; at this stage, we have an "initial" lattice $L_{2,0}$ whose edges are labeled by the elements of $P_{2}$. For syntactical reasons, we will often denote $\leq_{3}$ and $\leq_{2}$ by $\nu_{3}$ and $\nu_{2}$, respectively; for example, $\left\langle P_{3}, \nu_{3}\right\rangle=\left\langle P_{3}, \leq_{3}\right\rangle$. Letting

$$
\nu_{2,0}:=\operatorname{quo}\left(\nu_{3} \cup\left(\left\{0_{2}\right\} \times P_{2}\right) \cup\left(P_{2} \times\left\{1_{2}\right\}\right)\right),
$$

$\left\langle P_{2} ; \nu_{2,0}\right\rangle$ turns out to be a quasiordered set with bottom element $0_{2}=0_{3}$ and top element $1_{2}$. In fact, it is an ordered set; see (4.1) and (4.2). Since each edge of $L_{2,0}$ is either labeled, or thick and dotted (corresponding to the label $1_{3}$ ), or thick and solid (corresponding to $1_{2}$ ), or transposed to other edges in the same gadget, we can uniquely define a quasi-coloring

$$
\gamma_{0}^{*}: \text { Pairs } \leq\left(L_{2,0}\right) \rightarrow\left\langle P_{2} ; \nu_{2,0}\right\rangle
$$

analogously to (4.4). By construction, it is straightforward to see that $\gamma_{0}^{*}$ is a coloring and it extends $\gamma^{\prime}$; let us remind to (4.2) at this point. Let

$$
\left\{\left\langle u_{\iota}, v_{\iota}\right\rangle: 1 \leq \iota<\kappa\right\}:=\nu_{2} \backslash\left(\nu_{3} \cup\left\{\langle x, x\rangle: x \in P_{2}\right\} \cup\left\{\left\langle 0_{2}, x\right\rangle: x \in P_{2}\right\}\right)
$$

be the set of the comparabilities we intend to force by zigzag arrows; see (3.15) and (3.16). (Note that any smaller set whose union with $\nu_{3}$ generates $\nu_{2}$ as a quasiorder would do.) In (4.8), $\kappa$ is an ordinal number, that is, we have chosen a well-ordered index set. We let $\nu_{2, \iota}:=\operatorname{quo}\left(\nu_{2,0} \cup\left\{\left\langle u_{\mu}, v_{\mu}\right\rangle: 1 \leq \mu<\iota\right\}\right)$. By transfinite induction, we define lattices $L_{2, \iota}$, quasiordered (in fact, ordered) sets $\left\langle P_{2} ; \nu_{2, \iota}\right\rangle$ and quasi-colorings (in fact, colorings)

$$
\gamma_{\iota}^{*}: \text { Pairs } \leq\left(L_{2, \iota}\right) \rightarrow\left\langle P_{2} ; \nu_{2, \iota}\right\rangle
$$

in the following way. The case $\iota=0$ is settled by (4.6) and (4.7). If $\iota$ is a limit ordinal, then the ordering $\nu_{2, \iota}$ is the directed union of $\left\{\nu_{2, \mu}: \mu<\iota\right\}$. Let the lattice $L_{2, \iota}$ and the coloring $\gamma_{\iota}^{*}$ be the directed union of $\left\{L_{2, \mu}: \mu<\iota\right\}$ and that of $\left\{\gamma_{\mu}^{*}: \mu<\iota\right\}$, respectively; it is straightforward to see that $\gamma_{\iota}^{*}$ is a (quasi-) coloring. So the real task is to step from an ordinal $\iota$ to the next ordinal, $\mu:=\iota+1$. In order to accomplish this step, assume as an induction hypothesis that $\gamma_{\iota}^{*}$ from (4.9) is a coloring. Then $\nu_{\mu}=\operatorname{quo}\left(\nu_{\iota} \cup\left\{\left\langle u_{\iota}, v_{\iota}\right\rangle\right\}\right)$ and we obtain $L_{\mu}$ from $L_{\iota}$ by adding, from the $v_{\iota}$-colored basic edge $\left\langle a_{v_{\iota}}, b_{v_{\iota}}\right\rangle$ to the $u_{\iota}$-colored basic edge $\left\langle a_{u_{\iota}}, b_{u_{\iota}}\right\rangle$,

(A) a zigzag arrow if $u_{\iota} \in P_{3} \backslash P_{2}$, or

(B) a double-lined zigzag arrow if $u_{\iota} \in P_{2} \backslash\left\{0_{3}\right\}$. 
In both cases, the purpose of the arrow we add is to force the inequality in

$$
\gamma_{\mu}^{*}\left(\left\langle a_{u_{\iota}}, b_{u_{\iota}}\right\rangle\right)=\operatorname{con}\left(a_{u_{\iota}}, b_{u_{\iota}}\right) \leq \operatorname{con}\left(a_{v_{\iota}}, b_{v_{\iota}}\right)=\gamma_{\mu}^{*}\left(\left\langle a_{v_{\iota}}, b_{v_{\iota}}\right\rangle\right) .
$$

Hence, it is straightforward to check the validity of $(\mathrm{C} 1)$ for $\gamma_{\mu}^{*}$ as follows. Assume that $r:=\gamma_{\mu}^{*}\left(\left\langle x_{1}, x_{2}\right\rangle\right) \leq_{\nu_{\mu}} \gamma_{\mu}^{*}\left(\left\langle x_{3}, x_{4}\right\rangle\right)=: r^{\prime}$ for $\left\langle x_{1}, x_{2}\right\rangle,\left\langle x_{3}, x_{4}\right\rangle \in \operatorname{Pairs} \leq_{(}\left(L_{\mu}\right)$. Since $\nu_{\mu}=\operatorname{quo}\left(\nu_{\iota} \cup\left\{\left\langle u_{\iota}, v_{\iota}\right\rangle\right\}\right)$, there is a finite sequence $r=s_{0}, s_{1}, \ldots, s_{n}=r^{\prime}$ of elements in $P_{2}$ such that for each $i \in\{1, \ldots, n\}$,

$$
s_{i-1} \leq_{\nu_{\iota}} s_{i} \text { or }\left\langle s_{i-1}, s_{i}\right\rangle=\left\langle u_{\iota}, v_{\iota}\right\rangle \text {. }
$$

Since $\gamma_{\iota}^{*}$ : Pairs $\leq\left(L_{\iota}\right) \rightarrow P_{2}$ is a surjective map by the definition of quasi-colorings, we can pick pairs $\left\langle c_{i}, d_{i}\right\rangle \in \operatorname{Pairs} \leq\left(L_{\mu}\right)$ such that $\left\langle c_{0}, d_{0}\right\rangle=\left\langle x_{1}, x_{2}\right\rangle,\left\langle c_{n}, d_{n}\right\rangle=$ $\left\langle x_{3}, x_{4}\right\rangle, \gamma_{\iota}^{*}\left(\left\langle c_{i}, d_{i}\right\rangle\right)=s_{i}$ for all $i \in\{0, \ldots, n\}$, and, in addition, $\left\langle c_{i}, d_{i}\right\rangle=\left\langle a_{u_{\iota}}, b_{u_{\iota}}\right\rangle$ if $s_{i}=u_{\iota}$ and $\left\langle c_{i}, d_{i}\right\rangle=\left\langle a_{v_{\iota}}, b_{v_{\iota}}\right\rangle$ if $s_{i}=v_{\iota}$. Observe that for all $i \in\{1, \ldots, n\}$, we have that $\operatorname{con}_{L_{\mu}}\left(c_{i-1}, d_{i-1}\right) \leq \operatorname{con}_{L_{\mu}}\left(c_{i}, d_{i}\right)$ either because (4.11) and the validity of (C1) for $\gamma_{\iota}^{*}$, or because the (single-lined or double-lined) zigzag arrow forces that $\operatorname{con}_{L_{\iota}}\left(c_{i-1}, d_{i-1}\right)=\operatorname{con}_{L_{\iota}}\left(a_{u_{\iota}}, b_{u_{\iota}}\right) \leq \operatorname{con}_{L_{\iota}}\left(a_{v_{\iota}}, b_{v_{\iota}}\right)=\operatorname{con}_{L_{\iota}}\left(c_{i}, d_{i}\right)$. Therefore, by transitivity, we conclude that

$$
\operatorname{con}_{L_{\mu}}\left(x_{1}, x_{2}\right)=\operatorname{con}_{L_{\mu}}\left(c_{0}, d_{0}\right) \leq \operatorname{con}_{L_{\mu}}\left(c_{n}, d_{n}\right)=\operatorname{con}_{L_{\mu}}\left(x_{3}, x_{4}\right) .
$$

Thus, $\gamma_{\mu}^{*}$ satisfies $(\mathrm{C} 1)$.
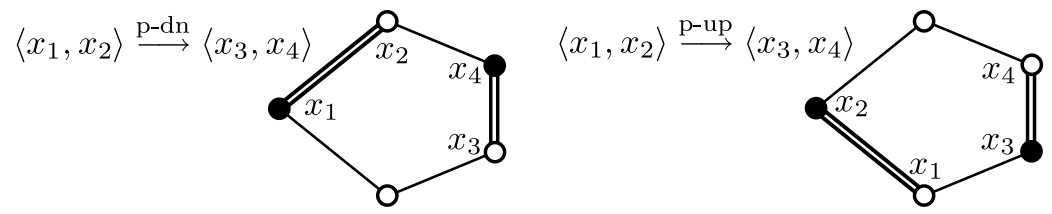

Figure 10. Prime perspectivities

Next, in order to prove the validity of $(\mathrm{C} 2)$ for $\gamma_{\mu}^{*}$, we begin with a weaker statement; namely, we are going to show that for every $x_{1}, \ldots, x_{4} \in L_{\mu}$,

$$
\begin{aligned}
& \text { if } x_{1} \prec x_{2}, x_{3} \prec x_{4}, \text { and } \operatorname{con}_{L_{\mu}}\left(x_{1}, x_{2}\right) \leq \operatorname{con}_{L_{\mu}}\left(x_{3}, x_{4}\right), \\
& \text { then } \gamma_{\mu}^{*}\left(\left\langle x_{1}, x_{2}\right\rangle\right) \leq_{\nu_{\mu}} \gamma_{\mu}^{*}\left(\left\langle x_{3}, x_{4}\right\rangle\right) \text {. }
\end{aligned}
$$

Following Grätzer [18] and using the definition given in Czédli, Grätzer, and Lakser [10], we say that the edge $\left\langle x_{1}, x_{2}\right\rangle$ is prime-perspective down to the edge $\left\langle x_{3}, x_{4}\right\rangle$, in notation $\left\langle x_{1}, x_{2}\right\rangle \stackrel{\mathrm{p}-\mathrm{dn}}{\longrightarrow}\left\langle x_{3}, x_{4}\right\rangle$, if $x_{2}=x_{1} \vee x_{4}$ and $x_{1} \wedge x_{4} \leq x_{3}$; see Figure 10, where the double-lined edges denote coverings, and observe the role of the black-filled elements in this definition. The upward prime perspectivity $\left\langle x_{1}, x_{2}\right\rangle \stackrel{\text { p-up }}{\longrightarrow}\left\langle x_{3}, x_{4}\right\rangle$ is defined dually. Since its proof uses induction on length, the Prime Projectivity Lemma of Grätzer [18] is valid for every lattice of finite length; this lemma asserts that $\operatorname{con}_{L_{\mu}}\left(x_{1}, x_{2}\right) \leq \operatorname{con}_{L_{\mu}}\left(x_{3}, x_{4}\right)$ if and only if there exists a finite sequence $\mathfrak{p}_{0}=\left\langle x_{3}, x_{4}\right\rangle, \mathfrak{p}_{1}, \ldots, \mathfrak{p}_{k-1}, \mathfrak{p}_{k}=\left\langle x_{1}, x_{2}\right\rangle$ of edges such that, for each $i \in\{1, \ldots, k\}$, $\mathfrak{p}_{i-1} \stackrel{\text { p-dn }}{\longrightarrow} \mathfrak{p}_{i}$ or $\mathfrak{p}_{i-1} \stackrel{\text { p-up }}{\longrightarrow} \mathfrak{p}_{i}$. Hence, by the transitivity of $\nu_{\mu}$, the required (4.12) would follow if we proved that

$$
\begin{aligned}
& \text { if }\left\langle x_{3}, x_{4}\right\rangle \stackrel{\text { p-dn }}{\longrightarrow}\left\langle x_{1}, x_{2}\right\rangle \text { or }\left\langle x_{3}, x_{4}\right\rangle \stackrel{\text { p-up }}{\longrightarrow}\left\langle x_{1}, x_{2}\right\rangle, \\
& \text { then } \gamma_{\mu}^{*}\left(\left\langle x_{1}, x_{2}\right\rangle\right) \leq_{\nu_{\mu}} \gamma_{\mu}^{*}\left(\left\langle x_{3}, x_{4}\right\rangle\right) .
\end{aligned}
$$


If $x_{1}, \ldots, x_{4} \in L_{\iota}$, then the premise of (4.13) gives $\operatorname{con}_{L_{\iota}}\left(x_{1}, x_{2}\right) \leq \operatorname{con}_{L_{\iota}}\left(x_{3}, x_{4}\right)$, whereby $\gamma_{\mu}^{*}\left(\left\langle x_{1}, x_{2}\right\rangle\right)=\gamma_{\iota}^{*}\left(\left\langle x_{1}, x_{2}\right\rangle\right) \leq_{\nu_{\mu}} \gamma_{\iota}^{*}\left(\left\langle x_{3}, x_{4}\right\rangle\right)=\gamma_{\mu}^{*}\left(\left\langle x_{3}, x_{4}\right\rangle\right)$ since $(\mathrm{C} 2)$ holds for $\gamma_{\iota}^{*}$ and $\nu_{\iota} \subseteq \nu_{\mu}$. So (4.13) holds if $x_{1}, \ldots, x_{4} \in L_{\iota}$. It also holds if $x_{1}, \ldots, x_{4}$ belong to the last added (single-lined or double-lined) zigzag arrow, because $\mathcal{G}_{2}$ is a colored lattice and $U_{p q}$ is isomorphic to $U_{p}$. We are left with the case where exactly one of the edges $\left\langle x_{1}, x_{2}\right\rangle$ and $\left\langle x_{3}, x_{4}\right\rangle$ belongs to Pairs $\leq\left(L_{\iota}\right)$. There are a lot of cases depending on the position of the edge not belonging to Pairs $\leq\left(L_{\iota}\right)$ but, similarly to Czédli [4], each of these cases can be settled in a straightforward way. Figures 8 and 9 reflect these cases satisfactorily but the rather tedious further details are omitted even if the present task based on the Prime Projectivity Lemma is slightly easier than the method used in Czédli [4]. After settling the above-mentioned cases, (4.13) follows, and it implies the validity of (4.12).

Next, for $\left\langle x_{1}, x_{2}\right\rangle,\left\langle x_{3}, x_{4}\right\rangle \in$ Pairs $\leq\left(L_{\mu}\right)$ that are not necessary edges, assume that $\operatorname{con}_{L_{\mu}}\left(x_{1}, x_{2}\right) \leq \operatorname{con}_{L_{\mu}}\left(x_{3}, x_{4}\right)$. Clearly, we can assume that $x_{1}<x_{2}$ and $x_{3}<x_{4}$. Let $x_{1}=y_{0} \prec y_{1} \prec \cdots \prec y_{m}=x_{2}$ and $x_{3}=z_{0} \prec z_{1} \prec \cdots \prec z_{n}=x_{4}$ be maximal chains in the corresponding intervals. The structure of $L_{\mu}$ makes it clear that in every chain of $L_{\mu}$, the set of $\gamma_{\mu}^{*}$-colors of the edges of this chain has a largest element. Hence, it follows from Czédli [4, Lemma 4.6], which says that (4.4) holds in every quasi-colored lattice with coloring map $\gamma^{\prime}$, that there are subscripts $i \in\{1, \ldots, m\}$ and $j \in\{1, \ldots, n\}$ such that

$$
\gamma_{\mu}^{*}\left(\left\langle x_{1}, x_{2}\right\rangle\right)=\gamma_{\mu}^{*}\left(\left\langle y_{i-1}, y_{i}\right\rangle\right) \text { and } \gamma_{\mu}^{*}\left(\left\langle x_{3}, x_{4}\right\rangle\right)=\gamma_{\mu}^{*}\left(\left\langle z_{j-1}, z_{j}\right\rangle\right) .
$$

Larger colors mean larger generated congruences since $\gamma_{\mu}^{*}$ satisfies $(\mathrm{C} 1)$. Using this fact together with (4.5), it follows that

$$
\operatorname{con}_{L_{\mu}}\left(x_{1}, x_{2}\right)=\operatorname{con}_{L_{\mu}}\left(y_{i-1}, y_{i}\right) \text { and } \operatorname{con}_{L_{\mu}}\left(x_{3}, x_{4}\right)=\operatorname{con}_{L_{\mu}}\left(z_{j-1}, z_{j}\right) .
$$

Combining (4.12), (4.14), and (4.15), it follows immediately that the assumption $\operatorname{con}_{L_{\mu}}\left(x_{1}, x_{2}\right) \leq \operatorname{con}_{L_{\mu}}\left(x_{3}, x_{4}\right)$ implies that $\gamma_{\mu}^{*}\left(\left\langle x_{1}, x_{2}\right\rangle\right) \leq_{\nu_{\mu}} \gamma_{\mu}^{*}\left(\left\langle x_{3}, x_{4}\right\rangle\right)$. Thus, $\gamma_{\mu}^{*}$ satisfies (C2). This completes the induction, whence we know that $\left\langle L_{\iota} ; \gamma_{\iota}^{*}, P_{2}, \nu_{\iota}\right\rangle$ is a quasi-colored lattice for all $\iota \leq \kappa$. In particular, $\left\langle L_{2} ; \gamma_{2}, P_{2}, \leq_{2}\right\rangle:=\left\langle L_{\kappa} ; \gamma_{\kappa}^{*}, P_{2}, \nu_{\kappa}\right\rangle$ is a colored lattice and the map $h_{2}:\left\langle P_{2} ; \leq_{2}\right\rangle \rightarrow\left\langle\operatorname{Princ}\left(L_{2}\right) ; \subseteq\right\rangle$ defined in (3.10) is an order isomorphism by (4.3). Finally, for $x \in P_{3}$,

$$
\begin{aligned}
\operatorname{Princ}\left(g_{3}\right)\left(h_{3}(x)\right) & \stackrel{(3.7)}{=} \operatorname{Princ}\left(g_{3}\right)\left(\operatorname{con}_{L_{3}}\left(a_{x}, b_{x}\right)\right) \stackrel{(1.1)}{=} \operatorname{con}_{L_{2}}\left(g_{3}\left(a_{x}\right), g_{3}\left(b_{x}\right)\right) \\
& \stackrel{(3.11)}{=} \operatorname{con}_{L_{2}}\left(a_{x}, b_{x}\right) \stackrel{(3.1)}{=} \operatorname{con}_{L_{2}}\left(a_{f_{3}(x)}, b_{f_{3}(x)}\right) \stackrel{(3.10)}{=} h_{2}\left(f_{3}(x)\right) .
\end{aligned}
$$

Hence, Princ $\left(g_{3}\right) \circ h_{3}=h_{2} \circ f_{3}$. Multiplying both sides by $h_{2}^{-1}$ from the left, we obtain that $f_{3}=h_{2}^{-1} \circ \operatorname{Princ}\left(g_{3}\right) \circ h_{3}$. This means that $g_{3}$ represents $f_{3}$ by means of principal congruences, completing the second proof of Lemma 4.2.

Now, we are in the position to complete the paper with two easy proofs as follows.

Proof of Theorem 1.2. Armed with Lemmas 4.1 and 4.2, the argument between (3.13) and Subsection 3.4 applies.

Proof of Remark 1.3. Let $M_{3,3,3,3}$ be one of the lattices that we can obtain from two copies of $M_{3,3}$ by means of a Hall-Dilworth gluing over a two-element intersection. If we replace $M_{3,3}$ by the simple lattice $M_{3,3,3,3}$ of length 5 , then our construction yields $L_{1}$ and $L_{2}$ such that they are of lengths 5 and 7 , respectively. 


\section{REFERENCES}

[1] Czédli, G.: Representing homomorphisms of distributive lattices as restrictions of congruences of rectangular lattices. Algebra Universalis 67, 313-345 (2012)

[2] Czédli, G.: A note on congruence lattices of slim semimodular lattices. Algebra Universalis, 72, 225-230 (2014)

[3] Czédli, G.: Representing a monotone map by principal lattice congruences. Acta Math. Hungar. 147, 12-18 (2015)

[4] Czédli, G.: The ordered set of principal congruences of a countable lattice. Algebra Universalis 75, 351-380 (2016)

[5] Czédli, G.: An independence theorem for ordered sets of principal congruences and automorphism groups of bounded lattices. Acta Sci. Math. (Szeged) 82, 3-18 (2016)

[6] Czédli, G.: Representing some families of monotone maps by principal lattice congruences. Algebra Universalis 77, 51-77 (2017)

[7] Czédli, G.: Cometic functors and representing order-preserving maps by principal lattice congruences. Algebra Universalis (to appear)

[8] Czédli, G.: Characterizing fully principal congruence representable distributive lattices. Algebra Universalis, DOI 10.1007/s00012-018-0498-8 (online April 12,2018)

[9] Czédli, G.: On the set of principal congruences in a distributive congruence lattice of an algebra. Acta Sci. Math. (Szeged) (to appear)

[10] Czédli, G., Grätzer, G., Lakser, H.: Congruence structure of planar semimodular lattices: the General Swing Lemma. Algebra Universalis, https://doi.org/10.1007/s00012-018-0483-2

[11] Czédli, G., Mureşan, C.: On principal congruences and the number of congruences of a lattice with more ideals than filters. Acta Sci. Math. (Szeged) (submitted), http://arxiv.org/abs/1711.06394

[12] Funayama, N., Nakayama, T.: On the distributivity of a lattice of lattice-congruences. Proc. Imp. Acad. Tokyo 18, 553-554 (1942)

[13] Grätzer, G.: Lattice Theory: Foundation. Birkhäuser Verlag, Basel (2011)

[14] Grätzer, G.: The order of principal congruences of a bounded lattice. Algebra Universalis 70, 95-105 (2013)

[15] Grätzer, G.: A technical lemma for congruences of finite lattices. Algebra Universalis 72, 53-55 (2014)

[16] Grätzer, G.: Planar semimodular lattices: congruences. In: Grätzer, G., Wehrung, F (eds) Lattice theory: special topics and applications. Vol. 1, pp. 131-165. Birkhäuser/Springer, Cham (2014)

[17] Grätzer, G.: Two more topics on congruence lattices of lattices. In: Grätzer, G., Wehrung, F (eds) Lattice theory: special topics and applications. Vol. 1, pp. 393-435. Birkhäuser/Springer, Cham (2014)

[18] Grätzer, G.: Congruences and prime-perspectivities in finite lattices. Algebra Universalis 74, 351-359 (2015)

[19] Grätzer, G.: The Congruences of a Finite Lattice. A Proof-by-picture Approach. Second edition. Birkhäuser, Basel (2016)

[20] Grätzer, G.: Homomorphisms and principal congruences of bounded lattices I. Isotone maps of principal congruences. Acta Sci. Math. (Szeged) 82, 353-360 (2016)

[21] Grätzer, G.: Homomorphisms and principal congruences of bounded lattices. II. Sketching the proof for sublattices. Algebra Universalis 78, 291-295 (2017)

[22] Grätzer, G.: Homomorphisms and principal congruences of bounded lattices. III. The Independence Theorem. Algebra Universalis 78 297-301 (2017)

[23] Grätzer, G.: Characterizing representability by principal congruences for finite distributive lattices with a join-irreducible unit element. Acta Math Szeged 83, 415-431 (2017)

[24] Grätzer, G., Knapp, E.: Notes on planar semimodular lattices. III. Congruences of rectangular lattices. Acta Sci. Math. (Szeged) 75, 29-48 (2009)

[25] Grätzer, G., Lakser, H.: Homomorphisms of distributive lattices as restrictions of congruences. Canad. J Math. 38, 1122-1134 (1986)

[26] Grätzer, G., Lakser, H.: Homomorphisms of distributive lattices as restrictions of congruences. II. Planarity and automorphisms. Canad. J. Math. 46, 3-54 (1994)

[27] Grätzer, G., Lakser, H.: Representing homomorphisms of congruence lattices as restrictions of congruences of isoform lattices. Acta Sci. Math. (Szeged) 75, 393-421 (2009) 
[28] Grätzer, G., Lakser, H.: Some preliminary results on the set of principal congruences of a finite lattice. Algebra Universalis 79: 21 (online, 2018) https://doi.org/10.1007/s00012-018-0487-y

[29] Grätzer, G., Lakser, H.: Minimal representations of a finite distributive lattice by principal congruences of a lattice. Acta Sci. Math. (Szeged), submitted

[30] Grätzer, G., Schmidt, E.T.: On congruence lattices of lattices. Acta Math. Acad. Sci. Hungar. 13, 179-185 (1962)

[31] Grätzer, G., Schmidt, E.T.: The strong independence theorem for automorphism groups and congruence lattices of finite lattices. Beiträge Algebra Geom. 36, 97-108 (1995)

[32] Huhn, A.P.: On the representation of algebraic distributive lattices. III. Acta Sci. Math. (Szeged) 53, 11-18 (1989)

[33] Nation, J. B.: Notes on Lattice Theory. http://www.math.hawaii.edu/ jb/books.html

[34] Růžička, P.: Free trees and the optimal bound in Wehrungs theorem. Fund. Math. 198, $217-228$ (2008)

[35] Schmidt, E. T.: The ideal lattice of a distributive lattice with 0 is the congruence lattices of a lattice. Acta Sci. Math. (Szeged) 43, 153-168 (1981)

[36] Wehrung, F.: A solution of Dilworth's congruence lattice problem. Adv. Math. 216, 610-625 (2007)

[37] Wehrung, F.: Schmidt and Pudlák's approaches to CLP. In: Grätzer, G., Wehrung, F (eds) Lattice theory: special topics and applications. Vol. 1, pp. 235-296. Birkhäuser/Springer, Cham (2014)

[38] Wehrung, F.: Congruence lattices and ideals of rings. In: Grätzer, G., Wehrung, F (eds) Lattice theory: special topics and applications. Vol. 1, pp. 297-335. Birkhäuser/Springer, Cham (2014)

[39] Wehrung, F.: Liftable and unliftable diagrams. In: Grätzer, G., Wehrung, F (eds) Lattice theory: special topics and applications. Vol. 1, pp. 337-392. Birkhäuser/Springer, Cham (2014)

E-mail address: czedli@math.u-szeged.hu

URL: http://www.math.u-szeged.hu/ ${ }^{\text {czedli/ }}$

University of Szeged, Bolyai Institute, Szeged, Aradi vértanúk tere 1, HUngary 6720 